\title{
The Overweight Challenge and Perceptions of Weight
}

\author{
Teresa Wills
}

School of Nursing and Midwifery, UCC, UCC

\section{Background}

Obesity is the most important health challenge faced at a global level and represents a rapidly growing problem to the health of populations. The prevalence of obesity is increasing worldwide and is an emerging healthcare epidemic affecting all populations. The World Health Organisation predicts that by 2015, 2.3 billion individuals will be overweight, and more than 700 million will be obese. Obesity is a relatively new phenomenon that has increased dramatically over the past three decades. In Ireland, adults are becoming overweight and obese at an alarming rate mirroring international patterns which signals a spiralling public health issue. Changing diets, consumption of high fat and high energy foods and increasingly sedentary lifestyles, have contributed to the generalised increase in body weight.

Obesity is a chronic disease and is characterised by an accumulation of excess body fat caused by increased caloric intake and decreased energy expenditure. Obesity is associated with complications that have a significant impact on health, psychosocial well-being, longevity, and quality of life for those affected. The rise in obesity rates have been accompanied by an increase in preventable chronic diseases. Eighty per cent of obese adults have at least one, and $40 \%$ have two, or more associated diseases such as diabetes, heart disease, gallbladder disease, breathing problems and certain forms of cancers (endometrial, breast, colon). Obesity appears to be responsible for a substantial economic burden and has a substantial impact on health care spending.

The increasing prevalence of obesity in Ireland has led to the normalisation of obesity within society. Recognition of the problem is a key component of obesity management and it remains especially crucial to address this issue. Reducing obesity is one of the greatest global public health challenges of the 21st century. Health care professionals have a key role to play in the prevention, treatment and management of obesity. Obesity cannot be cured, but effective prevention and treatment strategies are required and creating a lifestyle transformation for overweight and obese people must now become a major priority. 


\section{Perceptions of Weight}

As individuals become overweight or obese, they experience an increase in body image dissatisfaction which relates to an individual's ability to accurately perceive their body weight. Many people misperceive their weight and do not recognise their own overweight status. Inaccurate recognition of weight status by individuals is a threat to their health. The acceptance of higher body weights has led to individuals being unaware of the reality of their weight status and gravity of this situation. As individuals have become more tolerant of higher body weights, the need to perceive their weight status is delayed thus impeding recognition of obesity.

The decision to lose weight is heavily based on the perceptions of one's weight. Weight misperceptions among overweight and obese individuals may preclude adoption of healthy attitudes and behaviours. Awareness of personal weight status may be an important first step to avoid further weight gain. Having an adequate perception and interpretation of one's own body weight is the first step in maintaining a healthy body weight and prevention of being overweight. Encouraging behavioural changes can be difficult if an individual does not perceive that they are overweight or obese. The Health Belief Model represents a useful framework for understanding how individual's forms decisions that influence health behaviours and this framework will be used in the study to gain a deeper understanding of factors influencing weight loss.

Given the escalating global health problem of obesity, the need to re-appraise its management is more compelling than ever. It is widely accepted that the causes of obesity are complex and multi-factorial, therefore prevention demands an approach that enables a change of culture, attitudes and behaviour. In view of Ireland's increasing prevalence of obesity rates, a study to measure factors relating to motivation to change behaviour focusing on weight perceptions is timely.

\section{Aim}

The aim of the research was to determine factors relating to motivation to change behaviour in overweight individuals and to determine if the constructs of the Health Belief Model helped to explain motivation to change behaviour.

\section{Methods}

Following ethical approval, a descriptive, cross sectional, correlation study was undertaken. A sample of 202 men and women who perceived themselves to be overweight participated in the study. Data were collected using an online questionnaire. 


\section{Findings}

Results indicated a significant discrepancy between individual's perceptions of their body weight and their BMI-determined weight category. The findings showed that the majority of respondents (81\%) accurately perceived themselves to be overweight. Weight misperception was found in those who were obese with only $17 \%$ of respondents who were obese perceiving themselves to be obese. Over $60 \%$ of obese participants perceived themselves to be overweight and $21 \%$ perceived themselves to be very overweight. In contrast, most overweight respondents correctly perceived themselves to be overweight. The study results clearly show that the largest misperception was found in those who were obese.

\section{Conclusion and Recommendations}

The findings in this study offer insight into the need to examine the role of body weight perception in weight management strategies. Weight misperceptions are potentially modifiable, therefore public health strategies that promote physical activity and healthy eating behaviours need to focus on overcoming weight misperception. Greater routine monitoring and interpretation of body weight is required and could present an important step in managing the obesity epidemic. It is anticipated that the findings of this research will prompt health care professionals to focus on inaccurate weight perceptions in targeting weight loss effort among overweight and obese individuals. This should include routine monitoring and interpretation of body weight followed with education on enhanced benefits of modest weight loss and healthy lifestyles.

The finding draws attention to the need for health messages that challenge people to think about their weight as having an accurate perception on one's level of body weight can be a valuable first step in an individual's awareness of their weight status.

Teresa Wills is a College Lecturer and a DN student at the School of Nursing and Midwifery, UCC Cork under the supervision of Emeritus Professor Geraldine McCarthy and Dr Nicola Cornally, School of Nursing and Midwifery, Brookfield Health Sciences Complex, University College Cork., Ireland. 